\title{
Age Estimation with Panoramic Radiomorphometric Parameters Using Generalized Linear Models
}

\author{
Yeon-Hee Lee ${ }^{1}$, Jung-Sub $\mathrm{An}^{2}$ \\ 1Department of Orofacial Pain and Oral Medicine, Kyung Hee University Dental Hospital, Seoul, Korea \\ ${ }^{2}$ Department of Orthodontics, Seoul National University Dental Hospital, Seoul, Korea
}

Received February 18, 2021

Revised March 23, 2021

Accepted March 24, 2021
Correspondence to:

Yeon-Hee Lee

Department of Orofacial Pain and

Oral Medicine, Kyung Hee University

Dental Hospital, 26 Kyungheedae-ro,

Dongdaemun-gu, Seoul 02447, Korea

Tel: $+82-2-958-9454$

Fax: +82-2-958-9303

E-mail: omod0209@gmail.com

https://orcid.org/0000-0001-7323-0411
Purpose: The purpose of the present study was to investigate the correlation between age and 34 radiomorphometric parameters on panoramic radiographs, and to provide generalized linear models (GLMs) as a non-invasive, inexpensive, and accurate method to the forensic judgement of living individual's age.

Methods: The study included 417 digital panoramic radiographs of Korean individuals (178 males and 239 females, mean age: $32.57 \pm 17.81$ years). Considering the skeletal differences between the sexes, GLMs were obtained separately according to sex, as well as across the total sample. For statistical analysis and to predict the accuracy of the new GLMs, root mean squared error (RMSE) and adjusted R-squared $\left(R^{2}\right)$ were calculated.

Results: The adjusted $\mathrm{R}^{2}$-values of the developed GLMs in the total sample, and male and female groups were $0.623,0.637$, and 0.660 , respectively $(\mathrm{p}<0.001)$, while the allowable RMSE values were $8.80,8.42$, and 8.53 years, respectively. In the GLM of the total sample, the most influential predictor of greater age was decreased pulp area in the \#36 first molar (beta $=-26.52 ; p<0.01$ ), followed by the presence of periodontitis ( $b e t a=10.24 ; p<0.01$ ). In males, the most influential factor was the presence of periodontitis (beta $=9.20 ; p<0.05$ ), followed by the number of full veneer crowns (beta $=2.19 ; p<0.001$ ). In females, the most influential predictor was the presence of periodontitis (beta $=18.10 ; p<0.001)$, followed by the tooth area of the \#16 first molar (beta=-11.57; $\mathrm{p}<0.001$ ).

Conclusions: We established acceptable GLM for each sex and found out the predictors necessary to age estimation which can be easily found in panoramic radiographs. Our study provides reference that parameters such as the area of tooth and pulp, the number of teeth treated, and the presence of periodontitis should be considered in estimating age.

Key Words: Age estimation; Generalized linear model; Panoramic radiograph; Periodontitis; Pulp area; Tooth area

\section{INTRODUCTION}

Age estimation is an essential topic in forensic dentistry. It is necessary to identify the dead, but is also used in living individuals to clarify legal queries and to solve judicial or civil problems [1-3]. Although several body parts can be used to estimate age, burns, serious accidents, or decomposition often result in damage or loss of many body parts.
In such cases, dental age can often be used. It provides a better estimate of physiological age than skeletal or other common estimation methods [4]. Dental age estimation is relatively simple and accurate. It is not affected by environmental factors as it is predominantly determined by genetics, and the teeth are less affected by environmental factors than other body parts.

For estimating age, multiple indicators are present in

Copyright (c) 2021 Korean Academy of Orofacial Pain and Oral Medicine. All rights reserved.

(c) This is an open-access article distributed under the terms of the Creative Commons Attribution Non-Commercial License (http://creativecommons.org/licenses/by-nc/4.0/), which permits unrestricted non-commercial use, distribution, and reproduction in any medium, provided the original work is properly cited. 
skeletal and dental information. These factors can show broad variability in size, shape, and proportions across individuals [5]. As such, the accuracy of age estimation increases when multiple indicators are used, rather than a single parameter. Although each tooth can be used to determine age, the canines and first molars are particularly suitable candidates since they are commonly found at older ages, are less prone to dental caries, and have a larger root and pulp area than other teeth $[6,7]$. Therefore, canine and first molar have been frequently used for age estimation. However, no previous studies have comprehensively examined the use of canines and first molars together in this regard.

Changes in tooth shape and length, as well as increased numbers of treated and missing teeth, can also be predictors of greater age. Tooth surface loss is a macroscopically irreversible process that accumulates with age. Lambrechts et al. [8] estimated that the normal vertical loss of enamel due to physiological wear was approximately 20 to 38 $\mu \mathrm{m}$ per year [8]. When excessive tooth wear occurs, tooth shortening results, leading to exposed dentin, tooth hypersensitivity, or, more seriously, exposure of the canal, tooth hypersensitivity, pulpitis, and pulp necrosis [9]. The extent and severity of dental caries also increase with age, causing tooth loss. In the elderly, dental implant placement in edentulous areas is becoming more common, with the peak age for implant placement being between 60 and 75 years [10]. In summary, as age increases, the length and size of the teeth change, and the number of treated teeth, missing teeth, and dental implants may increase.

In addition to teeth, the mandible contains crucial structures, such as the mandibular canal (MC) and mental foramen. The anatomical location of the mental foramen may allow forensic scientists to determine the age and sex of an individual [11]. When mandibular teeth are lost, the adjacent alveolar bone resorbs to varying degrees [12,13]. Furthermore, many morphological and anatomical changes occur in the mandible with advancing age, and there are differences according to sex and dentoalveolar condition [14]. Unfortunately, the line demarcating normal from abnormal change blurs with advancing age; at times, this can make identifying pathological change more difficult in older adults.
Periodontitis occurs in all age groups, with a high prevalence of $25 \%$ to $47 \%$. However, the prevalence of periodontitis tends to increase over the age of 65 [15]. The most common oral manifestations of periodontitis are alveolar bone resorption and destruction [16]. The optimum method for assessing these characteristics of periodontitis is radiography [17]. However, the presence of periodontitis has not been comprehensively analyzed alongside other radiomorphometric parameters of the maxillofacial area that are used for age estimation.

Panoramic radiographs (PRs) are non-invasive and are a commonly used method of dental radiography. As such, they play a vital role in forensic dentistry to uncover hidden facts that cannot be seen using clinical or physical examination [18]. For these reasons, PRs are more accessible, more familiar socio-biologically, and have been extensively studied. The tendency of tooth pulp size to decrease with age can be easily investigated using PRs and confirmed by numerical values. Previous studies have used PRs to examine decreased pulp size with secondary and third dentin formation of the tooth, tooth loss, and periodontitis $[14,19]$. However, the present study was the first to comprehensively investigate all of these factors together.

Linear regression models have been adapted to estimate age using different dental parameters [20,21]. However, such models can only reliably estimate linear functions. If the relationship between input and output is non-linear, linear regression can only establish a local approximation. When used at a global level in such cases, the approximation can become inaccurate and distorted, even with the best linear approximation [22].

In contrast, generalized linear models (GLMs) are useful when there is no simple linear relationship between the input and output. As such, they are efficient under some not too-restrictive assumptions [23]. To evaluate model performance metrics with identity links under polynomial regressions, adjusted R-squared $\left(\mathrm{R}^{2}\right)$ and root mean squared error (RMSE) are commonly used. Higher $\mathrm{R}^{2}$ and lower RMSE values indicate better models. Age estimation has rarely been considered within the GLM framework, even though the GLM is one of the most commonly used statistical models in medical and clinical research nowadays because it can be used to obtain the sensitivities to all individual input 
factors at once.

The present study aimed to assess whether statistical GLMs could be exploited to estimate age using multiple radiomorphometric parameters in a non-destructive method. In addition, the predictive performance of the GLM with the training data set was investigated with a test data set.

We investigated the shape and length of the maxillary canine, the position of the MC, the position of the mental foramen, the pulp-to-tooth relationship of the upper and lower first molars, the number of treated teeth and missing teeth, and the presence of periodontitis. Furthermore, the present study was the first to comprehensively assess the various factors of the maxilla and mandible that change with increasing age. Specifically, we examined which factors are most related to age and whether there are sex differences. The study was conducted to assess the chronological age of Korean participants using skeletal and dental information in digital PRs. The aim was to derive a population-specific formula based on regression analysis to estimate age.

\section{MATERIALS AND METHODS}

The research protocol was in compliance with the Helsinki Declaration and was approved by the Institutional Review Board of the Kyung Hee University Dental Hospital (IRB no. KH-DT19018). Informed consent was obtained from all participants.

\section{Study Population and Design}

The sample consisted of 417 healthy patients (178 males and 239 females) with a known age of 11 to 70 years (mean age: $32.57 \pm 17.81$ years). All participants had PRs and were receiving dental care at Kyung Hee University Dental Hospital between April 1, 2018 and May 31, 2020. Multiple anatomical parameters in the maxilla and mandible were investigated on the patients' PRs. The inclusion criteria for patient selection were as follows: (1) all parameters clearly visible in the PR image; (2) permanent dentition; (3) full eruption of the selected maxillary canines and four first molars into the oral cavity in all quadrants; (4) root of selected canines and molars fully formed. None of the subjects had any developmental, endocrine, or nutritional disorders, and none had any special dental pathologies, such as amelogenesis imperfecta or dentinogenesis imperfecta. Patients with systemic disorders that can affect tooth maturation, eruption, and bone growth were excluded from the study, as were any teeth with developmental anomalies. Those with a history of maxillofacial, maxillary, or mandibular surgery were also excluded, as were those with mixed dentition.

PRs were acquired using an X-ray imaging machine (Promax 2D; Planmeca Oy, Helsinki, Finland), with the same distance between the film and the X-ray tube, beam angulation, film size, and exposure time used in all patients. The head position of the patients was maintained using a chin rest and bite guide. The optimal image density and contrast were achieved at exposure settings of $84 \mathrm{kVp}, 16$ $\mathrm{mA}$, and 16 seconds. The magnification factor was 1.20 . PR data were saved in Digital Imaging and Communications in Medicine (DICOM) files, while the Picture Archiving Communication System (PACS) was used to analyze the DICOM data, establish reference lines, and generate quantitative measurements. All measurements were performed using the utility tool in the tool bar of the PACS program, and each factor was measured bilaterally. Length was measured in $\mathrm{mm}$ and area in $\mathrm{cm}^{2}$. All measurement processes and investigations were conducted by two investigators (YHL and JSA). Internal consistency was represented using Cronbach's $\alpha$, by which all values were over $0.9(\mathrm{p}<0.001)$.

\section{Evaluations on Panoramic Radiography}

We investigated multiple parameters on the maxilla and mandible, including quantitative measurements and their ratios on the maxillary canines, maxillary and mandibular first molars, and the vertical positions of the MC and mental foramen. To determine the shape and length of the maxillary canine, crown length (CL), root length (RL), tooth length (TL), and crown-to-root ratio (CRR) were measured. To identify the pulp-to-tooth relationship of the upper and lower first molars, tooth area (TA), pulp area, and pulp-totooth area ratio (PTR) were investigated (Fig. 1).

\section{Length of the Upper Canine}

The CL (distance from the incisal to the alveolar bone crest $[\mathrm{AC}]$ ) and RL (distance from the AC to the apex) of the 


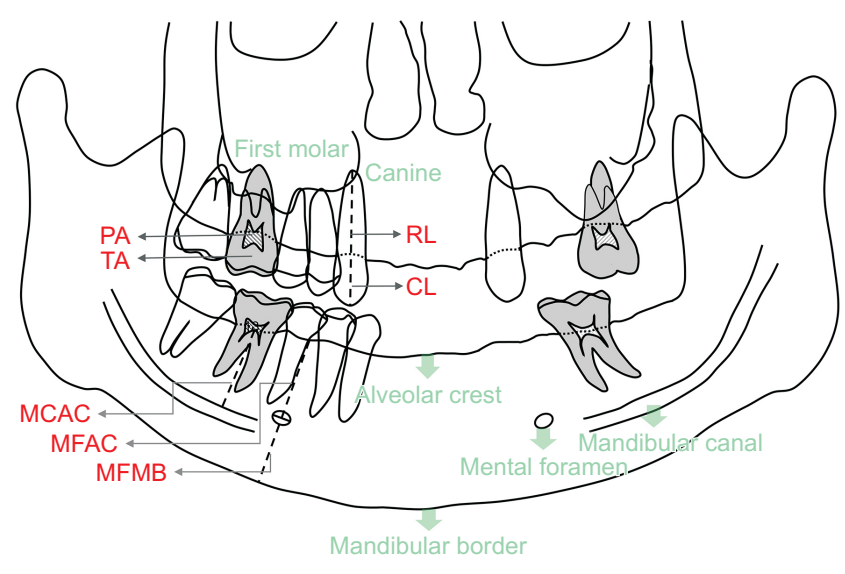

Fig. 1. Schematic representation of parameters measured on a panoramic radiograph. PA, pulp area of the maxillary and mandibular first molars; TA, tooth area of the maxillary and mandibular first molars; RL, root length of the maxillary canine; $\mathrm{CL}$, crown length of the maxillary canine; MCAC, distance from mandibular canal to alveolar crest on the mandibular first molar position; MFAC, distance from mental foramen to alveolar crest; $M F M B$, distance from mental foramen to mandibular border.

maxillary canine were measured on the longitudinal axis of the tooth, which extends incisocervically from the crown tip to the root apex. The TL (distance from the incisal to the apex) of the maxillary canine was measured by summing these two factors.

\section{Position of Mandibular Canal}

At the position of the mandibular first molar, a tangent line was drawn to the superior border of the MC. A vertical line was then drawn from the tangent line to the $\mathrm{AC}$ of the mandibular first molar. Next, the distance between the MC and $\mathrm{AC}$ (MCAC) was measured and the relative vertical position of the MC in the mandible was investigated.

\section{Position of Mental Foramen}

At the position of the mental foramen (MF), a tangent line was drawn to the inferior cortical outline of the mandible, and a vertical line was drawn in the direction of the AC. On that line, the sum of the distances from the MF to the $\mathrm{AC}$ (MFAC) and from the MF to the mandibular inferior border (MFMB) was defined as mandibular thickness (MT).

\section{Tooth and Pulp Area of the First Molar}

The whole tooth and pulp area of four maxillary and mandibular first molars were measured in the PR image of

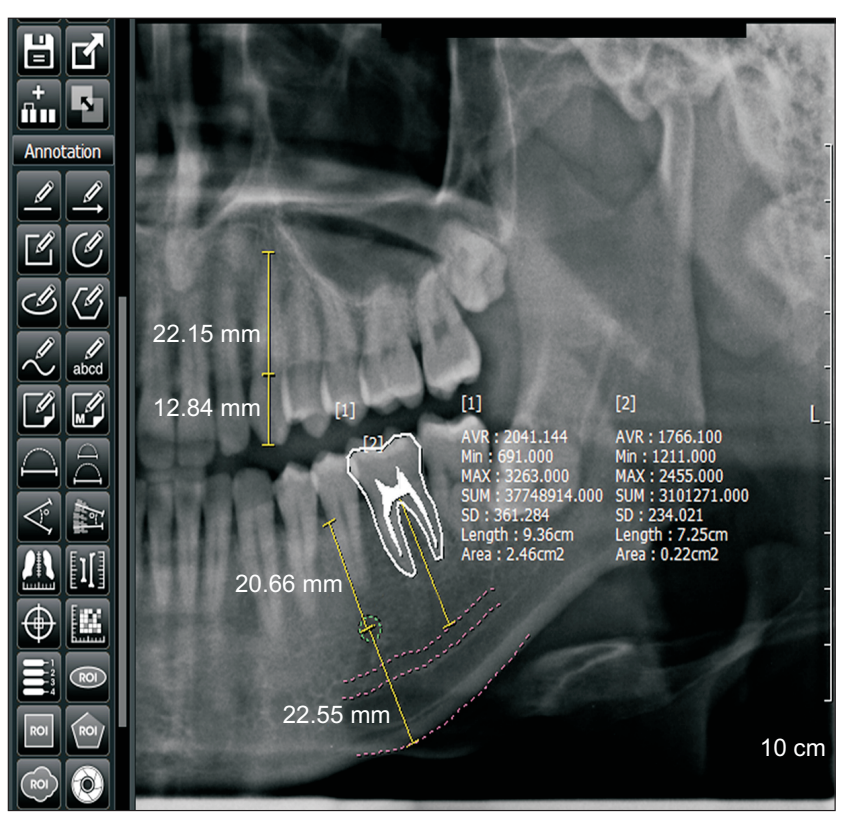

Fig. 2. Representative example of direct measurements on a panoramic radiograph.

each individual. To this end, a set of points was used to determine the entire tooth outlines. After completion of the tracing, the area in the frame was calculated automatically using the PACS program. Next, the outline of the pulp from the pulp chamber in the crown to root apex was traced, and the area of the pulp was measured automatically. Finally, the ratio of pulp area to total TA was determined (Fig. 2). We investigated changes in the whole TA, pulp area, and the pulp/ TA ratio with increasing age.

\section{Number of Missing and Treated Teeth}

We performed a visual assessment of the number of missing teeth, endodontically treated teeth, full veneer crowns, and implant prostheses. This process was conducted by two investigators. A total of 28 teeth (seven per quadrant) was set as the normal number from the incisors to the second molars; the third molars were excluded from the evaluation.

\section{Presence of Periodontitis}

Based on the distance from the alveolar bone level to the cemento-enamel junction, when alveolar bone destruction was observed at $\geq 30 \%$ of probing sites [24], we diagnosed periodontitis. To test the repeatability of the measurements, 30 patients were randomly re-evaluated 2 weeks after the initial measurements. The test-retest reliability for these 
analyses, represented using inter-class correlation (ICC), ranged from 0.92 to 0.99 , indicating excellent reliability.

\section{Statistical Analysis}

Descriptive statistics are reported as mean \pm standard deviations or numbers with percentages, where appropriate. Wilcoxon's rank-sum test was performed to compare the mean difference between males and females for each parameter. Pearson's correlation coefficient was calculated to investigate the linear relationship between age and each predictive parameter. Two statistical software programs, IBM SPSS Statistics (Version 22.0; IBM, Armonk, NY, USA) and R (Version 4.0.2; R Foundation for Statistical Computing, Vienna, Austria), were used for all statistical analysis. A two-tailed p-value of $<0.05$ was considered statistically significant. All measurements and investigations were conducted by two investigators. Internal consistency was represented using Cronbach's $\alpha$, and test-retest reliability was represented using ICC.

To establish the GLMs, we randomly divided all subjects into the training data set and the test set at a ratio of 8:2. The GLMs were established using the training data set, and a validation test of the model was performed using the test data set. Network weights were derived using the training set and the model performance tested on the test set. The data in the training and test sets recorded the actual age of the subjects, so the predicted and real ages could be compared and the predictive performance of the model assessed. Discrepancies between the predicted and observed ages were assessed in each test and training dataset. To quantify predictive performance, the RMSE was utilized. The RMSE measures the average error of the model when predicting the outcome of an observation; it was defined as follows:

$$
\text { RMSE }=\sqrt{\frac{\sum(\text { Real age-Predicted age })^{2}}{n}}
$$

To evaluate model performance metrics, the adjusted $R^{2}$ value was used. It is a powerful tool to select variables in GLMs that have identity links in polynomial regressions. Specifically, $\mathrm{R}^{2}$ is the proportion of variation in outcome that is explained by the predictor variables. In multiple regression models, $\mathrm{R}^{2}$ is the square of the correlation between the observed outcome values and the values predicted by the model. Higher $\mathrm{R}^{2}$-values indicate better models.

\section{RESULTS}

\section{Correlation Analysis}

The descriptive statistics of radiomorphometric parameters used to develop the GLMs are shown in Table 1. The CL of the maxillary canine was longer, the MT thicker, the TA of the first molars larger, and the prevalence of periodontitis higher in males than in females. Since the difference in skeleton size between men and women also appears in the maxillofacial region, we analyzed males and females separately, in addition to the overall data analysis.

The Pearson's correlation coefficients between age and all measured parameters in all participants are presented in Table 2. A negative correlation coefficient indicates that the magnitude of these parameters decreased with age. In the maxillary canine, the CL increased with age, and the RL decreased. This pattern was found in the total sample, as well as in males and females, with the strongest correlation occurring in females.

For MCAC, a negative correlation coefficient occurred across in the total sample, as well as in males and females, indicating that the MCAC decreased with age. MFMB showed a positive correlation with age in the total sample, as well as in males and females. This means that the mandibular foramen moves away from the mandibular inferior border as age increases. There was significant negative correlation between age and PA in the maxillary and mandibular first molars in the total sample, as well as in males and females, and the magnitude of mandibular first molars was greater than that of maxillary first molars. There was no correlation between age and the TA of the maxillary or mandibular first molars. The PTR of the first molars showed a significant negative correlation with age in the total sample, as well as in males and females. The magnitude of the correlation was greater in the mandible than in the maxilla. In females and in the total sample, the number of missing teeth showed a positive correlation with age. In in the total sample, as well as in males and females, the number of implant prostheses, endodontically treated teeth, and full veneer crowns was significantly positively correlated with age, indicating that the number of dental treated teeth increased 
Table 1. Descriptive statistics of radiomorphometric parameters

\begin{tabular}{|c|c|c|c|c|c|}
\hline \multicolumn{2}{|c|}{ Parameter } & Total & Male & Female & $p$-value \\
\hline \multicolumn{6}{|l|}{ Maxillary canine } \\
\hline \multirow[t]{4}{*}{ Maxillary right canine } & CL_13 & $9.85 \pm 2.03$ & $10.10 \pm 2.18$ & $9.68 \pm 1.90$ & $0.0095^{\star \star}$ \\
\hline & RL_13 & $17.80 \pm 3.13$ & $18.38 \pm 3.21$ & $17.37 \pm 3.01$ & $0.0027 * *$ \\
\hline & TL_13 & $27.66 \pm 3.89$ & $28.49 \pm 3.96$ & $27.05 \pm 3.73$ & $0.0004^{* * *}$ \\
\hline & CRR_13 & $56.98 \pm 15.40$ & $56.60 \pm 15.98$ & $57.26 \pm 14.97$ & 0.8294 \\
\hline \multirow[t]{4}{*}{ Maxillary left canine } & $\mathrm{CL}_{-} 23$ & $9.76 \pm 2.14$ & $10.06 \pm 2.44$ & $9.541 \pm 1.85$ & $0.0068^{* *}$ \\
\hline & RL_23 & $17.92 \pm 3.20$ & $18.57 \pm 3.16$ & $17.44 \pm 3.14$ & $0.0011 * *$ \\
\hline & TL_23 & $27.68 \pm 3.90$ & $28.63 \pm 3.99$ & $26.97 \pm 3.68$ & $0.0002^{\star * *}$ \\
\hline & CRR_23 & $56.47 \pm 18.45$ & $55.96 \pm 18.34$ & $56.84 \pm 18.57$ & 0.5985 \\
\hline \multicolumn{6}{|c|}{ Mental foramen and mandibular canal } \\
\hline \multirow{2}{*}{ Mandibular canal } & MCAC_36 & $23.84 \pm 4.40$ & $24.84 \pm 4.59$ & $23.10 \pm 4.11$ & $<0.001 * \star \star *$ \\
\hline & MCAC_46 & $23.71 \pm 4.37$ & $24.83 \pm 4.77$ & $22.87 \pm 3.86$ & $<0.001^{* * *}$ \\
\hline \multirow[t]{3}{*}{ Right mental foramen } & MFMB_Rt & $14.99 \pm 2.71$ & $15.92 \pm 2.77$ & $14.31 \pm 2.46$ & $<0.001 * * *$ \\
\hline & MFAC_Rt & $20.29 \pm 3.98$ & $20.98 \pm 4.03$ & $19.78 \pm 3.88$ & $0.0016 * *$ \\
\hline & BT_Rt & $35.29 \pm 5.37$ & $36.90 \pm 5.45$ & $34.09 \pm 4.99$ & $<0.001 * \star *$ \\
\hline \multirow[t]{3}{*}{ Left mental foramen } & MFMB_Lt & $14.97 \pm 2.65$ & $15.75 \pm 2.71$ & $14.40 \pm 2.46$ & $<0.001 * * \star$ \\
\hline & MFAC_Lt & $20.00 \pm 3.65$ & $20.86 \pm 3.72$ & $19.36 \pm 3.48$ & $<0.001 * * *$ \\
\hline & BT_Lt & $34.98 \pm 5.11$ & $36.61 \pm 5.07$ & $33.76 \pm 4.79$ & $<0.001 * * *$ \\
\hline \multicolumn{6}{|c|}{ Pulp and tooth area of first molar } \\
\hline \multirow[t]{2}{*}{ Maxillary right first molar } & TA_16 & $1.79 \pm 0.45$ & $1.88 \pm 0.46$ & $1.73 \pm 0.43$ & $0.0043^{* *}$ \\
\hline & PA_16 & $0.11 \pm 0.05$ & $0.11 \pm 0.05$ & $0.11 \pm 0.05$ & 0.1341 \\
\hline \multirow[t]{2}{*}{ Maxillary left first molar } & TA_26 & $1.81 \pm 0.43$ & $1.88 \pm 0.41$ & $1.76 \pm 0.44$ & $0.0029 * *$ \\
\hline & PA_26 & $0.11 \pm 0.05$ & $0.11 \pm 0.05$ & $0.11 \pm 0.05$ & 0.1190 \\
\hline \multirow[t]{2}{*}{ Mandibular left first molar } & TA_36 & $1.99 \pm 0.44$ & $2.05 \pm 0.44$ & $1.95 \pm 0.44$ & $0.0274^{*}$ \\
\hline & PA_36 & $0.18 \pm 0.08$ & $0.18 \pm 0.08$ & $0.18 \pm 0.08$ & 0.5695 \\
\hline \multirow[t]{2}{*}{ Mandibular right first molar } & TA_46 & $1.94 \pm 0.46$ & $2.02 \pm 0.47$ & $1.89 \pm 0.45$ & $0.0104 *$ \\
\hline & PA_46 & $0.17 \pm 0.08$ & $0.18 \pm 0.08$ & $0.17 \pm 0.08$ & 0.5818 \\
\hline \multirow[t]{4}{*}{ Pulp to tooth area ratio } & PTR_16 & $6.36 \pm 2.64$ & $6.25 \pm 2.44$ & $6.45 \pm 2.78$ & 0.7110 \\
\hline & PTR_26 & $6.06 \pm 2.66$ & $6.05 \pm 2.63$ & $6.07 \pm 2.68$ & 0.8314 \\
\hline & PTR_36 & $9.12 \pm 3.93$ & $9.02 \pm 3.88$ & $9.20 \pm 3.97$ & 0.7031 \\
\hline & PTR_46 & $8.91 \pm 3.68$ & $8.59 \pm 3.91$ & $9.15 \pm 3.49$ & 0.1073 \\
\hline \multicolumn{6}{|l|}{ Intra-oral inspection } \\
\hline \multirow[t]{6}{*}{ No. of teeth } & Total & $26.94 \pm 1.80$ & $26.82 \pm 2.01$ & $27.02 \pm 1.63$ & 0.2393 \\
\hline & Missing & $0.86 \pm 1.69$ & $0.99 \pm 1.94$ & $0.77 \pm 1.48$ & 0.2169 \\
\hline & Implant prosthesis & $0.34 \pm 1.05$ & $0.43 \pm 1.30$ & $0.27 \pm 0.82$ & 0.3320 \\
\hline & Endodontically treated & $1.01 \pm 2.12$ & $0.99 \pm 2.03$ & $1.03 \pm 2.16$ & 0.6580 \\
\hline & Full veneer crown prosthesis & $1.70 \pm 3.12$ & $1.90 \pm 3.43$ & $1.54 \pm 2.86$ & 0.6126 \\
\hline & Periodontitis & $0.12 \pm 0.33$ & $0.17 \pm 0.38$ & $0.08 \pm 0.28$ & $0.0087 * *$ \\
\hline
\end{tabular}

$\overline{\mathrm{CL}}$, crown length of the maxillary canine; $\mathrm{RL}$, root length of the maxillary canine; $\mathrm{TL}$, tooth length of the maxillary canine; $\mathrm{CRR}$, crown length to root length ratio of maxillary canine; 13 , maxillary right canine; 23, maxillary left canine; MCAC, the distance from mandibular canal to alveolar crest on mandibular first molar position; 36, mandibular left first molar; 46, mandibular right first molar; MFMB, the distance from mental foramen to mandibular border; MFAC, the distance from mental foramen to alveolar crest; BT, mandibular bone thickness; Rt, right side; Lt, left side; TA, tooth area of the maxillary and mandibular first molars; PA, pulp area of maxillary and mandibular first molars; 16 , maxillary right first molar; 26, maxillary left first molar; PTR, pulp area to tooth area ratio of the maxillary mandibular first molars.

Values are presented as mean \pm standard deviation. Results were obtained via t-test.

$p$-value was considered as significant when $p$-value $<0.05\left({ }^{*} p<0.05,{ }^{* *} p<0.01,{ }^{* *} p<0.001\right)$.

with age. In addition, there was a significant positive correlation between age and the presence of periodontitis.

\section{The Developed Generalized Linear Models}

The prediction performance of the developed models in the test and training sets is presented in Table 3. Considering the adjusted $\mathrm{R}^{2}$-value, the performance of the developed GLMs in the training set was acceptable in all groups (total sample, male, and female; adjusted $\mathrm{R}^{2}$ ranging from 0.623 to $0.660 ; p<0.001$ in all cases) (Fig. 3). With 
Table 2. Pearson's correlation coefficient between age and measured variables

\begin{tabular}{|c|c|c|c|c|}
\hline \multicolumn{2}{|c|}{ Parameter } & \multirow{2}{*}{$\frac{\text { Total }(n=417)}{0.365^{* *}}$} & \multirow{2}{*}{$\frac{\text { Male }(n=178)}{0.327^{\star \star}}$} & \multirow{2}{*}{$\frac{\text { Female }(n=239)}{0.405^{\star *}}$} \\
\hline Maxillary canine & $\mathrm{CL}_{-} 13$ & & & \\
\hline & $\mathrm{RL} \_13$ & $-0.203^{\star *}$ & $-0.175^{\star \star}$ & $-0.265^{\star \star}$ \\
\hline & TL_13 & 0.027 & 0.025 & 0.008 \\
\hline & CRR_13 & $0.421 * *$ & $0.383^{* *}$ & $0.473^{* *}$ \\
\hline & $\mathrm{CL}_{2} 23$ & $0.336^{* *}$ & $0.320 * *$ & $0.351 * *$ \\
\hline & $\mathrm{RL}_{-} 23$ & $-0.223^{* *}$ & $-0.212^{\star \star}$ & $-0.271 * *$ \\
\hline & TL_23 & 0.001 & -0.019 & 0.000 \\
\hline & CRR_23 & $0.376 * \star$ & $0.351 * *$ & $0.415^{\star *}$ \\
\hline \multirow[t]{2}{*}{ Mandibular canal } & MCĀC_36 & $-0.157 * *$ & $-0.163^{*}$ & $-0.182^{*}$ \\
\hline & MCAC_46 & $-0.147^{* *}$ & $-0.157^{*}$ & $-0.173^{*}$ \\
\hline \multirow[t]{6}{*}{ Mental foramen } & MFMB_Rt & $0.197 * *$ & $0.130^{*}$ & $0.262^{* *}$ \\
\hline & MFAC_Rt & 0.074 & 0.049 & 0.084 \\
\hline & BT_Rt & $0.155^{* *}$ & 0.103 & $0.202^{* *}$ \\
\hline & MFMB_Lt & $0.212^{* *}$ & $0.141 *$ & $0.285^{\star \star}$ \\
\hline & MFAC_Lt & 0.032 & 0.013 & 0.038 \\
\hline & BT_Lt & $0.131 * \star$ & 0.080 & $0.173 *$ \\
\hline \multirow[t]{8}{*}{ Pulp and tooth area } & TA_16 & -0.047 & -0.105 & 0.027 \\
\hline & PA_16 & $-0.157 * \star$ & $-0.147^{*}$ & $-0.178^{*}$ \\
\hline & TA_26 & -0.030 & -0.048 & -0.013 \\
\hline & PA_26 & $-0.238^{* *}$ & $-0.261 * *$ & $-0.207 * *$ \\
\hline & TA_36 & -0.050 & -0.096 & -0.004 \\
\hline & PA_36 & $-0.361 * *$ & $-0.374^{\star \star}$ & $-0.347 * \star$ \\
\hline & TA_46 & -0.048 & -0.067 & -0.041 \\
\hline & PA_46 & $-0.401 * *$ & $-0.382^{* *}$ & $-0.430 \star *$ \\
\hline \multirow[t]{4}{*}{ Pulp to tooth area ratio } & PTR_16 & $-0.170 * \star$ & $-0.140 *$ & $-0.219 * *$ \\
\hline & PTR_26 & $-0.267 * *$ & $-0.291 * *$ & $-0.233^{* *}$ \\
\hline & PTR_36 & $-0.374^{* *}$ & $-0.368^{\star *}$ & $-0.381 * *$ \\
\hline & PTR_46 & $-0.440 * *$ & $-0.401 * *$ & $-0.484^{\star *}$ \\
\hline \multirow[t]{6}{*}{ Intra-oral inspection } & Total teeth & 0.024 & 0.119 & -0.075 \\
\hline & Missing teeth & $0.108^{*}$ & 0.027 & $0.188^{*}$ \\
\hline & Implant prosthesis & $0.408^{* *}$ & $0.453^{* *}$ & $0.385^{\star \star}$ \\
\hline & Endodontically treated teeth & $0.435^{\star *}$ & $0.426^{\star *}$ & $0.445^{\star \star}$ \\
\hline & Full veneer crown prosthesis & $0.606 * \star$ & $0.605^{\star *}$ & $0.610 * \star$ \\
\hline & Periodontitis & $0.589 * *$ & $0.543^{* *}$ & $0.642 * *$ \\
\hline
\end{tabular}

$\mathrm{CL}$, crown length of the maxillary canine; $\mathrm{RL}$, root length of the maxillary canine; $\mathrm{TL}$, tooth length of the maxillary canine; $\mathrm{CRR}$, crown length to root length ratio of maxillary canine; 13 , maxillary right canine; 23 , maxillary left canine; MCAC, the distance from mandibular canal to alveolar crest on mandibular first molar position; 36, mandibular left first molar; 46, mandibular right first molar; MFMB, the distance from mental foramen to mandibular border; MFAC, the distance from mental foramen to alveolar crest; BT, mandibular bone thickness; Rt, right side; Lt, left side; TA, tooth area of the maxillary and mandibular first molars; PA, pulp area of maxillary and mandibular first molars; 16, maxillary right first molar; 26, maxillary left first molar; PTR, pulp area to tooth area ratio of the maxillary mandibular first molars.

Results were obtained using Pearson's correlation analysis.

$p$-value was considered as significant when $p$-value $<0.05\left({ }^{*} p<0.05, * * p<0.01\right)$.

Table 3. Prediction performance of the developed GLMs in the training and test sets

\begin{tabular}{llccc}
\hline \multicolumn{1}{c}{ Parameter } & Total $(\mathrm{n}=417)$ & Male $(\mathrm{n}=178)$ & Female $(\mathrm{n}=239)$ \\
\hline \multirow{2}{*}{ Training set } & p-value of GLM & $<0.001 * * \star$ & $<0.001 * * \star$ & $<0.001 * * *$ \\
& Adjusted $\mathrm{R}^{2}$ & 0.623 & 0.637 & 0.660 \\
\multirow{3}{*}{ Test set } & Root mean square error & 8.80 & 8.42 & 8.53 \\
& Real age on the test set & $27.97 \pm 15.30$ & $28.36 \pm 16.59$ & $27.69 \pm 14.54$ \\
& Predicted age using GLM & $25.67 \pm 10.97$ & $26.28 \pm 10.91$ & $25.74 \pm 12.07$ \\
& Difference between real age and predicted age & $8.71 \pm 6.90$ & $8.12 \pm 6.11$ & $8.67 \pm 7.31$ \\
\hline
\end{tabular}

GLM, generalized linear model; $R^{2}, R$-squared value.

Values are presented as mean \pm standard deviation.

The results were obtained via generalized linear model using $R$ statistics.

$p$-value was considered as significant when $p$-value $<0.05(* * * p<0.001)$. 

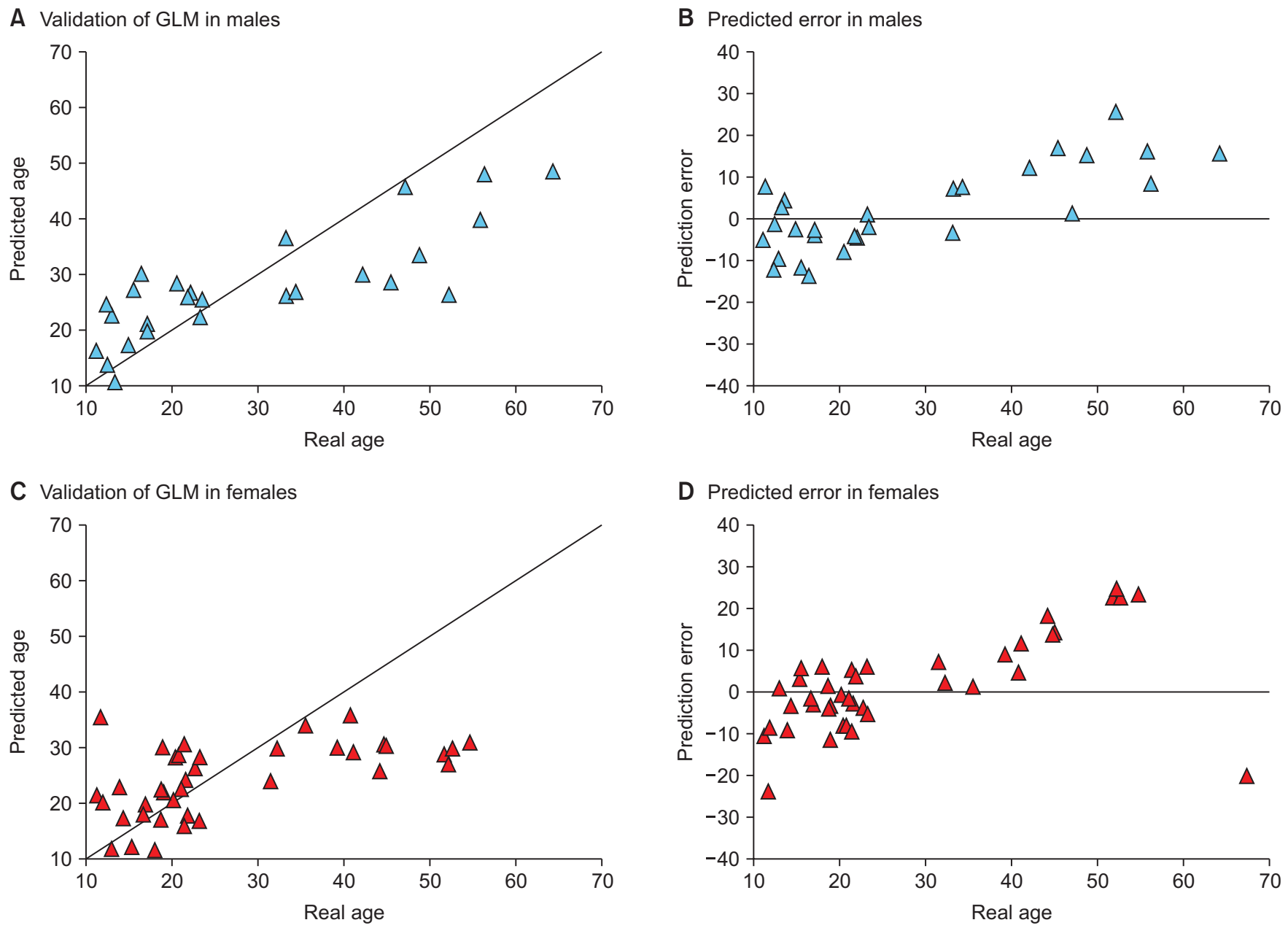

Fig. 3. Validation of the generalized linear models (GLMs) and predicted error between real and predicted age.

regards to the prediction error, based on the RMSE value for the training set, it was better to obtain separate models for males and females than to establish a model for the total sample. When applying each criterion of the GLM to the test set, the mean difference between the true and predicted ages was less than 10 years for all criteria. Table 3 and Fig. 1 compare prediction performance of the developed models in different sex groups in the test set.

The subjects' age was modeled as a function of the morphological variables (predictors), and to optimize the model, a stepwise linear regression analysis was performed to find these statistically significant predictors of chronological age.

\section{Total Sample}

The results show that the following variables contributed significantly to the fit: CL of the right maxillary canine (13),
RL of the left maxillary canine (23), CL to RL ratio of 23, MFMB of the left side, TA of the right (16) and left maxillary first molars (26), PA of the left mandibular first molar (36), PTR of the left maxillary (26) and right mandibular first molars (46), total number of teeth (TN), number of dental crown prostheses (CN), and presence of periodontitis, yielding the following GLM formula:

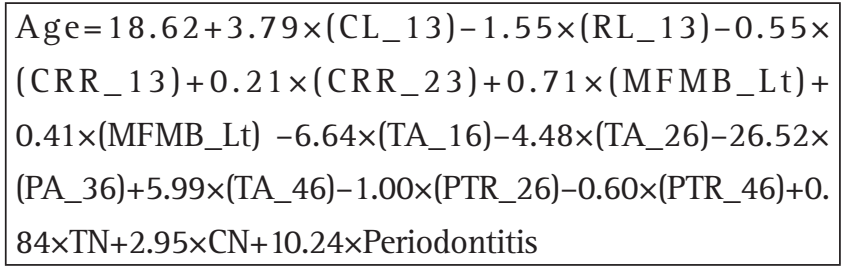

The adjusted $\mathrm{R}^{2}$ of the developed GLM for the total sample was acceptable $(0.623 ; \mathrm{p}<0.001)$, with an RMSE of 8.80 years. The difference between the true and predicted ages in the test set was $8.71 \pm 6.90$ years. In the GLM of the total 
sample, age was most closely associated with decrease in pulp area of the first molar (36; beta $=-26.52 ; \mathrm{p}<0.01)$, followed by the presence of periodontitis (beta $=10.24 ; \mathrm{p}<0.01$ ).

\section{Male}

The results show that the following variables contributed significantly to the fit: CL of the 13, RL of the 13, MCAC of 36 and 46 position, MFMB and MFAC of the left side, TA of 16, PTR of 16 and 36, TN, CN, and presence of periodontitis, yielding the following GLM formula:

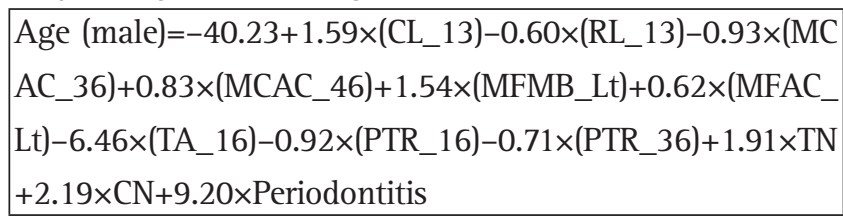

The adjusted $\mathrm{R}^{2}$-value of the developed GLM for the male sample was acceptable $(0.637 ; p<0.001)$, with an RMSE of 8.42 years. The difference between true and predicted age in the test set was $8.12 \pm 6.11$ years. In the GLM of the male sample, age was most closely associated with the presence of periodontitis (beta $=9.20 ; p<0.05$ ), followed by the number of full veneer crowns (beta $=2.19, p<0.001$ ).

\section{Female}

The following variables contributed significantly to the fit: CL of the 13, RL of the 13, CRR of the 13 and 23, TA of the 16 and 46, PA of the 36, PTR of the 26 and 46, distance from inferior alveolar nerve canal to alveolar crest at 36 and 46, MFMB and MFAC of the left side, TA of 16, PTR of 16 and 36, TN, IN, CN, and presence of periodontitis, yielding the following GLM formula:

Age (female $)=63.57+5.08 \times\left(C_{-} 13\right)-1.83 \times\left(R_{-}+13\right)-$ $0.86 \times\left(C R R \_13\right)+0.29 \times\left(C R R \_23\right)-11.57 \times\left(T A \_16\right)-$ $22.95 \times($ PA_36) $+7.24 \times($ TA_46) $-1.11 \times($ PTR_26) 0.77 $\times\left(\mathrm{PTR} \_46\right)+4.34 \times \mathrm{IN}+3.85 \times \mathrm{CN}+18.10 \times$ Periodontitis

The adjusted $\mathrm{R}^{2}$-value of the developed GLM for the male sample was acceptable $(0.660 ; p<0.001)$, with an RMSE 8.53 years and a difference of $8.67 \pm 7.31$ years between true and predicted age in the test set. In the GLM of the female sample, age was most closely associated with the presence of periodontitis (beta $=18.10 ; p<0.001$ ), followed by the tooth area of the first molar ( 16 ; beta $=-11.57 ; \mathrm{p}<0.001)$. The GLM for females estimated age better than that for males, with a larger adjusted $\mathrm{R}^{2}$, although the difference in performance between the sexes was negligible.

\section{DISCUSSION}

The present study aimed to find parameters associated with age that could be identified on PRs, as well as to provide a regression equation for age estimation using a GLM. Unlike a simple t-test or linear and logistic regression, GLMs can analyze more complicated relationships and ascertain the simultaneous effects of multiple variables, even mixtures of categorical and continuous variables [25]. In the GLMs of all categories, the average error was less than 10 years, and the range was 8.42 to 8.80 . In addition, the RMSE was used to measure the accuracy of prediction, ranging from 8.80 to 8.92 . Errors less than \pm 10 years are considered acceptable in forensic age prediction [26]. Therefore, the developed prediction model can be considered a useful tool to estimate age in forensic odontology.

$\mathrm{PR}$ is the most common and basic imaging method in dentistry and forensic odontology worldwide. It can visualize the entire mouth, including the teeth and surrounding bony structures, in one image. Computed tomography (CT) or cone beam CT is an ideal and accurate method for measuring PTR. However, installation of the machine is difficult, the method is expensive, and it exposes patients to X-rays [6]. On the contrary, PR has low cost and only uses a small dose of ionizing radiation, and it is a representative and non-invasive imaging technique [27]. Thus, PR has been used successfully in several studies to estimate age. In those studies, linear regression models were obtained using several tooth parameters as input variables [28-30]. Interestingly, the present study was the first to use PR to comprehensively investigate the age-related trends in multiple biometric features of the maxilla and mandible, including teeth and the vital structures of the mandible.

Genetic factors result in significant sex-related differences in the skeleton. Ozturk et al. [12] reported that most craniofacial parameters were significantly larger in males than in females. However, few studies have derived equations from radiomorphometric parameters for males and females separately, even though sex-differences should be reflected in 
the age estimation equation. Therefore, our GLM and information are valuable for use in age estimation according to sex. Furthermore, for more accurate and elaborate estimation using GLMs, a training data set to establish the model and a test data set for measuring the model's performance are required [31]. Thus, instead of working with all of the data together, which is a major mistake made in some studies, we randomly partitioned the data into training and test sets for the GLMs. The equations obtained from the total sample, male, and female data sets were quite different; that is, the types of parameters that were associated with age differed among the groups, as did the degree of association. In our results, the adjusted $\mathrm{R}^{2}$-values of all GLMs ranged from 0.623 to 0.660 , which is an acceptable, highly significant result. Thus, our GLM could be used to interpret our model and make reliable predictions.

In the present study, the decrease in pulp area of left mandibular first molars was a powerful predictor of age in the GLM using the total sample. In addition, decreased PTR of the first molar was significantly correlated with increased age. The size of the dental pulp cavity is reduced as a result of secondary dentin deposit, and measurements of this reduction can be used to indicate age. Gustafson [32] described age-related morphological changes, including attrition, secondary dentin, and cementum apposition in the dental tissues. Since 2004, many researchers have evaluated whether variations in pulp chamber size and PTR ratio is an indicator of age. This method of age estimation seems promising in canines and molars on PRs [33-36]. While those authors obtained acceptable levels of accuracy in age prediction (mean error: 3-9 years), they advised that future research investigate the effect of race and culture on the parameters.

The presence of periodontitis was the strongest predictor in our total sample and the second most influential factor in males and females. In people 65 years old and above, the prevalence of periodontitis tends to increase, regardless of region or race [37]. However, periodontitis is more prevalent in older age groups and seniors because of the greater adaptive immune response, reduced diversity, and enhanced levels of Porphyromonas gingivalis [38]. The prevalence of periodontitis is very high (14\%-65\%) and the condition is observed at all age groups [37,39]. However, the prevalence increases with age and the causes are complex. For example, the prevalence of periodontitis is much higher in those living below the poverty line and in those with less than a high school education [40]. Therefore, when considering periodontitis, the subject's socioeconomic status should be considered in addition to their oral hygiene. In addition, severe and chronic periodontitis can easily lead to tooth loss. The following parameters were significant predictors of tooth loss at baseline: number of teeth with restorations, mean probing pocket depth score, age, tobacco use, alcohol consumption, number of teeth present, and male sex [41]. Therefore, more information on the subject's oral condition and socioeconomic factors may be needed to clearly interpret our results.

An increased number of full veneer crowns was a powerful predictor of age in our male sample. The normal vertical loss of enamel from physiological wear is approximately 20 to $38 \mu \mathrm{m}$ per annum [8]. Jayawardena et al. [42] reported a negative correlation between age and CL over the alveolar bone level, indicating that TL and area are important in age determination. The percentage of adult patients presenting with severe tooth wear increased from 3\% at the age of 20 years to $17 \%$ at the age of 70 years, indicating a tendency to develop wear with age [43]. Throughout life, the teeth and other surrounding tissues in the mouth experience mechanical and chemical wear and damage due to daily mastication. Accordingly, the number of teeth requiring dental treatment increases with age, as does the number of dental implant prostheses. Of course, the varying life experiences of individuals can substantially affect the appearance, composition, resilience, and strength of teeth, so changes in the teeth and dentition of older adults can encompass a broad spectrum. However, identifying the general trend of dental treatment according to age is important to forensic dentistry.

Our research had some limitations. To ensure our results have clearer, more meaningful interpretation, further research should involve more participants. In addition, studies involving new technologies such as artificial intelligence may be necessary to allow the automatic identification of large amounts of PR data, as well as to identify and interpret all relevant factors in PRs. Furthermore, our subjects were only Asians, namely South Koreans, so we cannot 
generalize our interpretation to the other races of the world. Consequently, our study should be replicated using a wider population in which race and other relevant factors such as socioeconomic and nutritional status are taken into account.

The present study suggested that a new tool could be developed using GLM to predict age based on dental findings. Several radiomorphological parameters on PR were correlated with age in the GLM. Furthermore, there were sex differences in the size and length of anatomical factors, so sex-specific GLMs are needed. Thus, GLMs are useful for age estimation and should be used in forensic dentistry. As such, they should be explored further as more data become available and complementary research should be carried out to improve this method.

\section{CONFLICT OF INTEREST}

No potential conflict of interest relevant to this article was reported.

\section{ORCID}

\author{
Yeon-Hee Lee \\ https://orcid.org/0000-0001-7323-0411 \\ Jung-Sub An \\ https://orcid.org/0000-0002-2160-3295
}

\section{REFERENCES}

1. Juneja M, Devi YB, Rakesh N, Juneja S. Age estimation using pulp/tooth area ratio in maxillary canines-A digital image analysis. J Forensic Dent Sci 2014;6:160-165.

2. Mathew DG, Rajesh S, Koshi E, Priya LE, Nair AS, Mohan A. Adult forensic age estimation using mandibular first molar radiographs: a novel technique. J Forensic Dent Sci 2013;5:56-59.

3. Ilayaraja V, Ganapathy N, Jisha G, Keerthipriyadharshini T, Maheswaran T, Yoithapprabhunath TR. Digitized morphometric analysis using maxillary canine and mandibular first molar for age estimation in South Indian population. Open Dent J 2018;12:762769.

4. Manjunatha BS, Soni NK. Estimation of age from development and eruption of teeth. J Forensic Dent Sci 2014;6:73-76.

5. Fieuws S, Willems G, Larsen-Tangmose S, Lynnerup N, Boldsen J, Thevissen P. Obtaining appropriate interval estimates for age when multiple indicators are used: evaluation of an ad-hoc pro- cedure. Int J Legal Med 2016;130:489-499.

6. Farhadian M, Salemi F, Saati S, Nafisi N. Dental age estimation using the pulp-to-tooth ratio in canines by neural networks. Imaging Sci Dent 2019;49:19-26.

7. Panchbhai AS. Dental radiographic indicators, a key to age estimation. Dentomaxillofac Radiol 2011;40:199-212.

8. Lambrechts P, Braem M, Vuylsteke-Wauters M, Vanherle G. Quantitative in vivo wear of human enamel. J Dent Res 1989;68:17521754.

9. Donachie MA, Walls AW. The tooth wear index: a flawed epidemiological tool in an ageing population group. Community Dent Oral Epidemiol 1996;24:152-158.

10. Eklund SA. Trends in dental treatment, 1992 to 2007. J Am Dent Assoc 2010;141:391-399.

11. Kanchan T, Krishan K. Mental foramen in prediction of age. J Clin Diagn Res 2015;9:GJ01.

12. Ozturk CN, Ozturk C, Bozkurt M, Uygur HS, Papay FA, Zins JE. Dentition, bone loss, and the aging of the mandible. Aesthet Surg J 2013;33:967-974.

13. Lavelle CL. Preliminary study of mandibular shape after tooth loss. J Prosthet Dent 1985;53:726-730.

14. Okşayan R, Asarkaya B, Palta N, Şimşek İ, Sökücü O, İşman E. Effects of edentulism on mandibular morphology: evaluation of panoramic radiographs. ScientificWorldJournal 2014;2014: 254932.

15. Dye BA. Global periodontal disease epidemiology. Periodontol 2000 2012;58:10-25.

16. Hienz SA, Paliwal S, Ivanovski S. Mechanisms of bone resorption in periodontitis. J Immunol Res 2015;2015:615486.

17. Tugnait A, Carmichael F. Use of radiographs in the diagnosis of periodontal disease. Dent Update 2005;32:536-538, 541-542.

18. Saxena S, Sharma P, Gupta N. Experimental studies of forensic odontology to aid in the identification process. J Forensic Dent Sci 2010;2:69-76.

19. Dosi T, Vahanwala S, Gupta D. Assessment of the effect of dimensions of the mandibular ramus and mental foramen on age and gender using digital panoramic radiographs: a retrospective study. Contemp Clin Dent 2018;9:343-348.

20. Limdiwala PG, Shah JS. Age estimation by using dental radiographs. J Forensic Dent Sci 2013;5:118-122.

21. Shah PH, Venkatesh R. Pulp/tooth ratio of mandibular first and second molars on panoramic radiographs: an aid for forensic age estimation. J Forensic Dent Sci 2016;8:112.

22. Pianosi F, Beven K, Freer J, Hall JW, Rougier J, Stephenson DB. Sensitivity analysis of environmental models: a systematic review with practical workflow. Environ Model Softw 2016;79:214-232.

23. Lu R, Wang D, Wang M, Rempala GA. Estimation of Sobol's sensitivity indices under generalized linear models. Commun Stat Theory Methods 2018;47:5163-5195.

24. Renvert S, Persson RE, Persson GR. Tooth loss and periodontitis in older individuals: results from the Swedish National Study on Aging and Care. J Periodontol 2013;84:1134-1144.

25. Van Horn ML, Jaki T, Masyn K, et al. Evaluating differential effects using regression interactions and regression mixture models. Educ Psychol Meas 2015;75:677-714. 
26. Babshet M, Acharya AB, Naikmasur VG. Age estimation in Indians from pulp/tooth area ratio of mandibular canines. Forensic Sci Int 2010;197:125.e1-4.

27. Hwang SY, Choi ES, Kim YS, Gim BE, Ha M, Kim HY. Health effects from exposure to dental diagnostic X-ray. Environ Health Toxicol 2018;33:e2018017.

28. Dehghani M, Shadkam E, Ahrari F, Dehghani M. Age estimation by canines' pulp/tooth ratio in an Iranian population using digital panoramic radiography. Forensic Sci Int 2018;285:44-49.

29. Roh BY, Lee WJ, Ryu JW, Ahn JM, Yoon CL, Lee SS. The application of the Kvaal method to estimate the age of live Korean subjects using digital panoramic radiographs. Int J Legal Med 2018;132:1161-1166.

30. Lee JH, Lee C, Battulga B, et al. Morphological analysis of the lower second premolar for age estimation of Korean adults. Forensic Sci Int 2017;281:186.e1-186.e6.

31. Scheinost D, Noble S, Horien C, et al. Ten simple rules for predictive modeling of individual differences in neuroimaging. Neuroimage 2019;193:35-45.

32. Gustafson G. Age determination on teeth. J Am Dent Assoc 1950; 41:45-54.

33. Paewinsky E, Pfeiffer H, Brinkmann B. Quantification of secondary dentine formation from orthopantomograms--a contribution to forensic age estimation methods in adults. Int J Legal Med 2005;119:27-30.

34. Cameriere R, Brogi G, Ferrante L, et al. Reliability in age determination by pulp/tooth ratio in upper canines in skeletal remains. $\mathrm{J}$ Forensic Sci 2006;51:861-864.
35. Cameriere R, Ferrante L, Cingolani M. Precision and reliability of pulp/tooth area ratio (RA) of second molar as indicator of adult age. J Forensic Sci 2004;49:1319-1323.

36. Kim S, Lee YH, Noh YK, Park FC, Auh QS. Age-group determination of living individuals using first molar images based on artificial intelligence. Sci Rep 2021. doi: 10.1038/s41598-020-801828. [Epub ahead of print]

37. Demmer RT, Papapanou PN. Epidemiologic patterns of chronic and aggressive periodontitis. Periodontol 2000 2010;53:28-44.

38. Wu Y, Dong G, Xiao W, et al. Effect of aging on periodontal inflammation, microbial colonization, and disease susceptibility. J Dent Res 2016;95:460-466.

39. Costa FO, Guimarães AN, Cota LO, et al. Impact of different periodontitis case definitions on periodontal research. J Oral Sci 2009;51:199-206.

40. Borrell LN, Beck JD, Heiss G. Socioeconomic disadvantage and periodontal disease: the Dental Atherosclerosis Risk in Communities study. Am J Public Health 2006;96:332-339.

41. Copeland LB, Krall EA, Brown LJ, Garcia RI, Streckfus CF. Predictors of tooth loss in two US adult populations. J Public Health Dent 2004;64:31-37.

42. Jayawardena CK, Abesundara AP, Nanayakkara DC, Chandrasekara MS. Age-related changes in crown and root length in Sri Lankan Sinhalese. J Oral Sci 2009;51:587-592.

43. Van't Spijker A, Rodriguez JM, Kreulen CM, Bronkhorst EM, Bartlett DW, Creugers NH. Prevalence of tooth wear in adults. Int J Prosthodont 2009;22:35-42. 